\title{
HAUSDORFF DIMENSION DISTRIBUTION OF QUASICONFORMAL MAPPINGS ON THE HEISENBERG GROUP
}

\author{
By \\ ZOLTÁN M. BALOGH
}

\begin{abstract}
We construct quasiconformal mappings on the Heisenberg group which change the Hausdorff dimension of Cantor-type sets in an arbitrary fashion. On the other hand, we give examples of subsets of the Heisenberg group whose Hausdorff dimension cannot be lowered by any quasiconformal mapping. For a general set of a certain Hausdorff dimension we obtain estimates of the Hausdorff dimension of the image set in terms of the magnitude of the quasiconformal distortion.
\end{abstract}

\section{Statement of results}

It is a subject of general interest to understand the way a certain class of mappings changes the Hausdorff dimension of sets. It is well-known that topological mappings can change the Hausdorff dimension in an arbitrary fashion, while bilipschitz mappings do not change the dimension at all. For the class of quasiconformal (QC) mappings, the situation is much more subtle. The first results in this direction are due to Gehring and Väisälä [GeVa], and the subject is still under active research [As], [Bi], [BiTy1],[BiTy2], [Ty].

In the present paper, we study this problem for the class of $\mathrm{QC}$ mappings of the Heisenberg group. The Heisenberg group $H^{1}$ has underlying space $\mathbf{R}^{3}$ but is equipped with a metric $d_{H}$ that is very different from the usual Euclidean metric. This makes the study of Hausdorff dimension of sets more complicated than in the Euclidean case. We refer to the survey article of Gromov [Gr] for more details on the Heisenberg geometry. Basic definitions that we need in the sequel are recalled at the beginning of Section 2 .

Recall that a homeomorphism $f: H^{1} \rightarrow H^{1}$ is $K$-quasiconformal for some $K \geq 1$ if its quasiconformal distortion $H(x, f)$ is uniformly bounded on $H^{1}$ :

$$
H(x, f):=\lim \sup _{r \rightarrow 0} \frac{\sup _{d_{H}(x, y)=r} d_{H}(f x, f y)}{\inf _{d_{H}(x, y)=r} d_{H}(f x, f y)} \leq K, \quad \text { for all } x \in H^{1}
$$


A homeomorphism $f: H^{1} \rightarrow H^{1}$ is QC by this definition if it is $K$-QC for some $K \geq 1$.

QC mappings play a central role in many important problems, such as the quasiisometry classification of negatively curved spaces [ $\mathrm{GrPa}],[\mathrm{Pa} 1]$ and Mostow-type rigidity results $[\mathrm{Mo}],[\mathrm{Pal}]$. The theory of QC maps on the Heisenberg group was initiated by Mostow [Mo] and subsequently developed in the work of Pansu [Pa l], and of Korányi and Reimann [KoRe1], [KoRe2]. These advances of the theory make it possible to address the question of Hausdorff dimension distortion. Here is our first result:

Theorem 1.1. For any two numbers $\alpha, \beta, 0<\alpha<\beta<4$, there are compact sets $S_{\alpha}, S_{\beta} \subset H^{1}$ and a quasiconformal mapping $f: H^{1} \rightarrow H^{1}$ such that $\operatorname{dim} S_{\alpha}=$ $\alpha, \operatorname{dim} S_{\beta}=\beta$ and $f S_{\beta}=S_{\alpha}$.

The corresponding statement in the Euclidean setting is due to Gehring and Väisälä (cf. [GeVa]). The proof of Theorem 1.1 is considerably harder than in the Euclidean case. Because of the complicated geometry of the Heisenberg metric, the construction of (even smooth) QC maps becomes a non-trivial task. Smooth QC maps were obtained in [KoRe1], [KoRe2] using flows of certain special vectorfields. A different way of constructing fiber-preserving QC maps appears in [CaTa], [Ta2] (see also [Ta1], [Ba] for a similar result for circle bundles). The proof of Theorem 1.1 is given in Sections 2 and 3. We apply the flow method of Korányi and Reimann, combined with a dynamical construction. The idea is based on a suggestion of Seppo Rickman [Ri].

In our next statement, we estimate the Hausdorff dimension of the image $f S$ for an arbitrary set $S$ under an arbitrary $K$-QC map $f: H^{1} \rightarrow H^{1}$. Let us introduce some notation. For a fixed $K \geq 1$, we denote by $\mathcal{F}\left(H^{1}, K\right)$ the collection of all $K$-QC maps $f: H^{1} \rightarrow H^{1}$.

For $f \in \mathcal{F}\left(H^{1}, K\right)$, we denote by $J_{f}$ the volume derivative of $f$ given by

$$
J_{f}(x)=\lim _{r \rightarrow 0} \frac{|f(B(x, r))|}{|B(x, r)|}, \quad \text { for a.e. } x \in X
$$

As in the Euclidean case (see [Ge]), it turns out that QC mappings on the Heisenberg group have a higher integrability property as shown in Section 4 of [KoRe2]. Namely, if $f \in \mathcal{F}\left(H^{1}, K\right)$, then $J_{f} \in L_{\text {loc }}^{r}$ for some $r>1$ independent of $f \in \mathcal{F}\left(H^{1}, K\right)$. The following quantity is called the exponent of integrability for the class $\mathcal{F}\left(H^{1}, K\right)$ :

$$
p=p\left(H^{1}, K\right)=\sup \left\{r>0: J_{f} \in L_{\text {loc }}^{r} \text { for any } f \in \mathcal{F}\left(H^{1}, K\right)\right\}
$$


Theorem 1.2. Let $f: H^{1} \rightarrow H^{1}$ be a $K$-quasiconformal mapping and let $A \subset H^{1}$ be a set with Hausdorff dimension $\operatorname{dim} A=\alpha, 0 \leq \alpha \leq 4$. Then

$$
\frac{4 \alpha(p-1)}{4 p-\alpha} \leq \operatorname{dim} f A \leq \frac{4 p \alpha}{4(p-1)+\alpha}
$$

where $p=p\left(H^{1}, K\right)$ is from (1.2).

Theorem 1.2 follows from Theorem 4.1 of Section 4 . Theorem 4.1 is a generalization of the corresponding statement from [GeVa] in the context of $Q$-regular metric spaces, where a weak $(1, q)$ Poincaré inequality holds for a certain $q<Q$ (cf. [HeKo]).

Our final result states that there are subsets of $H^{1}$ whose Hausdorff dimension cannot be lowered by QC maps.

Theorem 1.3. For any $\alpha, 1 \leq \alpha \leq 4$ there exists a compact set $S_{\alpha} \subset H^{1}$ with $\operatorname{dim} S_{\alpha}=\alpha$ such that $\operatorname{dim} f S_{\alpha} \geq \alpha$ for any $Q C$ mapping $f: H^{1} \rightarrow H^{1}$.

In the Euclidean setting, Theorem 1.3 was recently proved by Tyson [Ty]. The case $0<\alpha<1$ is unknown even in the Euclidean setting. For $1 \leq \alpha<3$, our proof is similar to the one in [Ty]. In the case $3 \leq \alpha<4$, the proof is different. An important ingredient in this case is Pansu's isoperimetric inequality [Pa2], [Gr]. The proof of Theorem 1.3 is given in Section 5 .

In the opposite direction, one can ask about raising the Hausdorff dimension of sets by QC mappings. There is a recent result of Bishop [Bi], saying that the dimension of any positive dimensional subset of $\mathbf{R}^{n}$ can be raised to be arbitrarily close to $n$ by QC maps of $\mathbf{R}^{n}$. It is likely that with the methods developed in this paper one can approach this problem in the Heisenberg setting as well.

Let us finally mention that we are working in the first Heisenberg group $H^{1}$, rather than $H^{n}$, only to avoid cumbersome notation. Versions of Theorem 1.1, Theorem 1.2 and Theorem 1.3 (for $1 \leq \alpha \leq 3$ and $2 n+1 \leq \alpha \leq 2 n+2$ ) are also valid in the setting of the general Heisenberg group $H^{n}, n \geq 1$.

\section{Acknowledgments}

It is my pleasure to thank Pierre Pansu, Martin Reimann and Seppo Rickman for their interest in this work and for many valuable conversations. I wish to thank also Steve Buckley and the referee for numerous suggestions for improving both the content and style of this paper. 


\section{Cantor-type sets with a given dimension}

In a general metric space $X$, the Hausdorff dimension of $A \subset X$ is defined by

$$
\operatorname{dim} A=\inf \left\{a>0: H_{a}(A)=0\right\}=\sup \left\{a \geq 0: H_{a}(A)=\infty\right\} .
$$

Here $H_{a}(A)$ is the $a$-dimensional Hausdorff (outer) measure of $A$ given by

$$
H_{a}(A)=\lim _{\epsilon \rightarrow 0} \inf _{\mathcal{B}} \sum_{B_{i} \in \mathcal{B}}\left(\operatorname{diam} B_{i}\right)^{a} .
$$

In the above relation, the inf is taken over all countable coverings $\mathcal{B}=\left(B_{i}\right)_{i}$ of the set $A$ by sets $B_{i}$ with diameter less than $\epsilon$.

For most of this paper, our metric space $X$ will be the first Heisenberg group $\left(H^{1}, *\right)$ whose underlying space is $\mathbf{R}^{3}$ with group operation given by

$$
\left(x_{1}, y_{1}, z_{1}\right) *\left(x_{2}, y_{2}, z_{2}\right)=\left(x_{1}+x_{2}, y_{1}+y_{2}, z_{1}+z_{2}+2\left(y_{1} x_{2}-y_{2} x_{1}\right)\right) .
$$

The Heisenberg distance of two points $q_{1}, q_{2} \in H^{1}$ is defined by

$$
d_{H}\left(q_{1}, q_{2}\right)=\left\|q_{1}^{-1} * q_{2}\right\|_{H}
$$

where the Heisenberg norm $\|q\|_{H}$ is given by

$$
\|q\|_{H}=\left(\left(x^{2}+y^{2}\right)^{2}+z^{2}\right)^{1 / 4}, \quad \text { for } q=(x, y, z) \in H^{1} .
$$

Let us denote by $|\cdot|$ the usual Euclidean volume measure in $\mathbf{R}^{3}$. Using (2.2) combined with translations and dilations (cf. (2.5) and (2.6) below) we see that there exists a constant $C \geq 1$ such that for an arbitrary ball $B(p, r), p \in H^{1}, 0<r \leq 1$ in the Heisenberg metric we have

$$
\frac{1}{C} r^{4} \leq|(B(p, r))| \leq C r^{4} .
$$

The Heisenberg group is of topological dimension 3, but as a metric space with the metric $d_{H}$ has Hausdorff dimension 4. This follows from (2.3). Moreover, (2.3) shows that $\left(H^{1}, d_{H}\right)$ is a 4-regular metric measure space. To illustrate the intricate structure of the Heisenberg geometry, let us notice that the $0 z$ axis has Hausdorff dimension 2, while the $0 x$ and $0 y$ axes are one-dimensional. Moreover, a smooth (Euclidean) surface has Hausdorff dimension 3 with respect to the Heisenberg metric (see [Gr]). The usual product formulae for Hausdorff dimension [Ma] in Euclidean spaces are also false in the Heisenberg setting. Because of these differences, we cannot use the Euclidean statements; and so we have to work out the proofs of our results from scratch. 
Before getting into details, let us mention that there is another metric on $H^{1}$, the so-called sub-Riemannian or Carnot-Carathéodory metric (see [Be], [Gr]). This is defined using the left-invariant vector-fields

$$
X=\frac{\partial}{\partial x}+2 y \frac{\partial}{\partial z}, \quad Y=\frac{\partial}{\partial y}-2 x \frac{\partial}{\partial z},
$$

which satisfy Hörmander's condition. This metric is perhaps more frequently used in the literature (cf. [Be], [Gr]) than $d_{H}$. Since the two metrics are bilipschitz equivalent (see [Ko], [BaMi]), we can work with $d_{H}$, which is more suitable for our calculations.

In this section, we start the proof of Theorem 1.1. We shall construct the sets $S_{\alpha}, S_{\beta}$ that appear in the statement. These sets will be invariant sets for certain conformal dynamical systems. The maps defining the dynamical system are either Heisenberg translations

$$
T_{q_{0}}: H^{1} \rightarrow H^{1}, \quad \text { given by } T_{q_{0}}(q)=q_{0} * q,
$$

or dilations

$$
\delta_{a}(q)=\left(a x, a y, a^{2} z\right), \quad \text { for } a>0, \quad q=(x, y, z) \in H^{1},
$$

or finite compositions of the above two.

It follows directly from the definition (1.1) that our maps from (2.5) and (2.6) are 1-quasiconformal (or conformal in our terminology). Note that the composition of a $K-\mathrm{QC}$ mapping with a translation or a dilation is again a $K-\mathrm{QC}$ mapping.

Let $B=\left\{q \in H^{1}:\|q\|_{H}<1\right\}$ be the open unit ball in $H^{1}$ centered at $0 \in H^{1}$. A ball $B\left(q_{0}, r\right)$ of radius $r>0$ and center $q_{0} \in H^{1}$ is of the form $B\left(q_{0}, r\right)=T_{q_{0}} \circ \delta_{r} B$. Observe that for any $r>0, q_{0} \in H^{1}$ we have the relation

$$
\delta_{r} \circ T_{q_{0}}=T_{\delta_{r} q_{0}} \circ \delta_{r}
$$

Relation (2.7) implies that the image $B^{\prime}$ of the unit ball $B$ under a finite composition of translations and dilations

$$
B^{\prime}=\left(T_{q_{01}} \circ \delta_{r_{1}} \circ \cdots \circ T_{q_{0 n}} \circ \delta_{r_{n}}\right) B
$$

is a ball $B(q, r)$ of radius $r=r_{1} \cdots r_{n}$ and center $q=q\left(q_{01}, \ldots, q_{0 n}, r_{1} \ldots, r_{n}\right)$.

Let $B_{i}=B\left(q_{i}, r\right), i=1, \ldots, N_{0}\left(N_{0} \geq 2\right)$, be disjoint balls of radius $r>0$ contained in $B$. Let $f_{i}=\delta_{1 / r} \circ T_{q_{i}^{-1}}: B\left(q_{i}, r\right) \rightarrow B$ be the associated conformal mappings and $f: \bigcup_{i} B_{i} \rightarrow B$ the generated dynamical system (such that $\left.f\right|_{B_{i}}=f_{i}$ ). Denote by $S=S(f)$ the invariant set of $f$ defined by the conditions $S \subset \cup_{i} B_{i}$, 
$f S=S=f^{-1} S$. To construct the set $S$, consider for each $n$ the preimages $S_{n}:=f^{-n} \bar{B}$, where $f^{-n} \bar{B}$ is the union

$$
S_{n}=\bigcup_{i_{1} \ldots i_{n}} B_{i_{1} \ldots i_{n}} \quad \text { and } \quad B_{i_{1} \ldots i_{n}}=\left(f_{i_{n}}^{-1} \circ \cdots \circ f_{i_{1}}^{-1}\right) \bar{B} .
$$

By our previous consideration, $S_{n}$ is a union of $N_{0}^{n}$ disjoint Heisenberg balls of radius $r^{n}$. Clearly $S_{n+1} \subset S_{n}$, and we obtain $S$ as

$$
S=\bigcap_{n} S_{n}=\bigcap_{n} f^{-n} \bar{B}
$$

Let $\alpha=\log N_{0} / \log (1 / r)$. Consider the covering of $S$ by the balls $B_{i_{1} \ldots i_{n}}$ and let $n \rightarrow \infty$. It follows that

$$
H_{\alpha}(S) \leq \lim _{n \rightarrow \infty} \sum_{i_{1} \ldots i_{n}}\left(\operatorname{diam} B_{i_{1} \ldots i_{n}}\right)^{\alpha}=\lim _{n \rightarrow \infty} N_{0}^{n}\left(2 r^{n}\right)^{\alpha}=2^{\alpha} .
$$

This shows that $\operatorname{dim} S \leq \alpha$.

For the reverse inequality $\operatorname{dim} S \geq \alpha$, we use the so-called mass distribution method (cf. [Fa] Chapter 9). Namely, we assign the mass $1 / N_{0}^{n}$ to each of the $N_{0}^{n}$ balls $B_{i_{1} \ldots i_{n}}$ and $n \geq 0$. This defines a mass distribution $\mu$ on the collection of balls

$$
\mathcal{B}=\left\{B_{i_{1}, \ldots i_{n}}: 1 \leq i_{1}, \ldots, i_{n} \leq N_{0}, n \geq 0\right\} .
$$

Moreover, $\mu$ can be extended to the Borel sets of $H^{1}$ by

$$
\mu(A)=\inf \left\{\sum_{i} \mu\left(B_{i}\right): A \subset \bigcup_{i} B_{i}, B_{i} \in \mathcal{B}\right\},
$$

where $i$ is a multi-index of the form $i=i_{1}, \ldots i_{n}$.

Then $\mu$ becomes a probability measure on $H^{1}$ supported on $S$ (cf. [Fa] Proposition 1.7), which clearly coincides with the above mass distribution on the collection of balls $B_{i}$. Moreover, by standard arguments (cf. [Fa] p. 119), it follows that $\mu$ is an $\alpha$-regular measure on $S$ : there exists $C \geq 1$ such that for any Heisenberg ball $B(p, \rho)$ with $p \in S, \rho \leq 1$ we have

$$
\frac{1}{C} \rho^{\alpha} \leq \mu(B(p, \rho)) \leq C \rho^{\alpha} .
$$

Now the upper estimate in (2.8) shows that if $\left(B_{j}\right)_{j}$ is an arbitrary countable covering of $S$ by balls with centers in $S$, then

$$
\sum_{j}\left(\operatorname{diam} B_{j}\right)^{\alpha} \geq \frac{1}{C} \sum_{j} \mu\left(B_{j}\right) \geq \frac{1}{C} \mu(S) \geq \frac{1}{C},
$$

which shows that $\operatorname{dim} S \geq \alpha$. 
In conclusion, we have constructed a Cantor set $S$ with

$$
\operatorname{dim} S=\frac{\log N_{0}}{\log (1 / r)}
$$

We now make the following

Claim. There exists $\delta>0$ such that for any $N \geq 1$, there are $N^{4}$ disjoint closed balls of radius $\delta / N$ contained in $B$.

Proof of Claim. We shall use the fact that the Heisenberg group $H^{1}$ is a 4-regular space expressed by relation (2.3).

Given $0<r<1 / 2$, let us denote by $K(r)$ the maximal number such that there are disjoint balls $\bar{B}\left(q_{1}, r\right), \ldots, \bar{B}\left(q_{K(r)}, r\right)$ contained in $B$. The maximality of $K(r)$ implies that

$$
B(0,1-r) \subset \bigcup_{i=1}^{K(r)} B\left(q_{i}, 5 r\right)
$$

and by (2.3) we have

$$
|B(0,1-r)| \leq \sum_{i=1}^{K(r)}\left|B\left(q_{i}, 5 r\right)\right| \leq K(r) C_{1} r^{4}
$$

which gives

$$
K(r) \geq \frac{1}{C_{2}} \frac{1}{r^{4}}
$$

Choosing

$$
\delta<\min \left\{\frac{1}{2}, \frac{1}{\left(C_{2}\right)^{1 / 4}}\right\} \text { and } r=\frac{\delta}{N}
$$

in relation $(2.10)$ the claim follows.

For arbitrary $0<a \leq 1$, consider the $N^{4}$ disjoint balls $B\left(q_{1}, a \delta / N\right), \ldots, B\left(q_{N^{4}}, a \delta / N\right)$ contained in $B$ whose existence is ensured by the Claim. Consider the associated dynamical system $f: \bigcup_{i=1}^{N^{4}} B\left(q_{i}, a \delta / N\right) \rightarrow B$ and its invariant set $S=S(f)$.

Formula (2.9) (with $N_{0}=N^{4}$ and $r=a \delta / N$ ) implies that $d(N, a)=\operatorname{dim} S$ is given by

$$
d(N, a)=\frac{4 \log N}{\log N-\log (a \delta)}
$$

Substituting $a=1$ in relation (2.11), we have

$$
d(N, 1)=\frac{4 \log N}{\log N-\log \delta} \rightarrow 4 \quad \text { as } N \rightarrow \infty .
$$


On the other hand, for $a=(1 / N)^{N}$ we obtain that

$$
d\left(N,(1 / N)^{N}\right) \rightarrow 0 \quad \text { as } N \rightarrow \infty
$$

This shows that for an arbitrary value $0<\alpha<4$ we can construct a Cantor set $S_{\alpha}$ as above such that $\operatorname{dim} S_{\alpha}=\alpha$.

\section{Maps distorting the Hausdorff dimension}

Proof of Theorem 1.1. Let us fix two values $\alpha, \beta$ such that $0<\alpha<\beta<4$. By our previous consideration, we can choose $N \geq 2$ and $r_{1}=r_{1}(N, \beta)$ such that there exist disjoint balls $B\left(q_{i}, r_{1}\right) \subset B, i=1, \ldots, N$ for which the associated conformal dynamical system $f: \bigcup_{i} B\left(q_{i}, r_{1}\right) \rightarrow B$ defined by

$$
\left.f\right|_{B\left(q_{i}, r_{1}\right)}=f_{i}=\delta_{1 / r_{1}} \circ T_{q_{i}^{-1}}: B\left(q_{i}, r_{1}\right) \rightarrow B
$$

has the invariant set $S=S_{\beta}$ where $\operatorname{dim} S_{\beta}=\beta$. Similarly, we can choose $r_{2}=$ $r_{2}(N, \alpha)<r_{1}$ such that the conformal dynamical system $g: \bigcup_{i} B\left(q_{i}, r_{2}\right) \rightarrow B$ defined by

$$
\left.g\right|_{B\left(q_{i}, r_{2}\right)}=g_{i}=\delta_{1 / r_{2}} \circ T_{q_{i}^{-1}}: B\left(q_{i}, r_{2}\right) \rightarrow B
$$

has the invariant set $S=S_{\alpha}$ where $\operatorname{dim} S_{\alpha}=\alpha<\beta$. We are going to construct a quasiconformal mapping $F: H^{1} \rightarrow H^{1}$ such that $F S_{\beta}=S_{\alpha}$. The mapping $F$ will satisfy $\left.F\right|_{H^{1} \backslash B}=\left.\mathrm{id}\right|_{H^{1} \backslash B}$. Inside the ball $B$, we define $F$ inductively using our dynamics $f: \bigcup_{i} B\left(q_{i}, r_{1}\right) \rightarrow B$ and $g: \bigcup_{i} B\left(q_{i}, r_{2}\right) \rightarrow B$ as follows.

Let $\epsilon>0$ be a small number (to be determined later), and consider the multiring

$$
A_{0}^{\epsilon}:=B(0,1+\epsilon) \backslash\left(\bigcup_{i} f_{i}^{-1} B(0,1-\epsilon)\right)
$$

and its iterated preimages $A_{(n)}^{\epsilon}:=f^{-n} A_{0}^{\epsilon}$,

$$
A_{(n)}^{\epsilon}=\bigcup_{i_{1} \ldots i_{n}} A_{i_{1} \ldots i_{n}}^{\epsilon} \quad \text { where } A_{i_{1} \ldots i_{n}}^{\epsilon}=f_{i_{n}}^{-1} \circ \cdots \circ f_{i_{2}}^{-1} \circ f_{i_{1}}^{-1} A_{0}^{\epsilon}
$$

Similarly, let

$$
\tilde{A}_{0}^{\epsilon}:=B(0,1+\epsilon) \backslash\left(\bigcup_{i} g_{i}^{-1} B(0,1-\epsilon)\right),
$$

and its corresponding iterated preimages $\tilde{A}_{(n)}^{\epsilon}:=g^{-n} \tilde{A}_{0}^{\epsilon}$,

$$
\tilde{A}_{(n)}^{\epsilon}=\bigcup_{i_{1} \ldots i_{n}} \tilde{A}_{i_{1} \ldots i_{n}}^{\epsilon} \quad \text { where } \tilde{A}_{i_{1} \ldots i_{n}}^{\epsilon}=g_{i_{n}}^{-1} \circ \cdots \circ g_{i_{2}}^{-1} \circ g_{i_{1}}^{-1} \tilde{A}_{0}^{\epsilon}
$$


In the first step of our construction, we define a smooth quasiconformal mapping $F_{0}: A_{0}^{\epsilon} \rightarrow \tilde{A}_{0}^{\epsilon}$ with the following properties:

(1) $F_{0}=$ id on $R_{0}^{\epsilon}=B(0,1+\epsilon) \backslash B\left(0, r_{1}(1-\epsilon)\right)$,

(2) $F_{0}=g_{i}^{-1} \circ f_{i}$ on $R_{i}^{\epsilon}:=f_{i}^{-1} R_{0}^{\epsilon}$.

Our first condition on the magnitude of $\epsilon>0$ is to guarantee

$$
R_{i}^{\epsilon} \cap R_{j}^{\epsilon}=\emptyset, \quad \text { for } 0 \leq i, j \leq N, \quad i \neq j
$$

so that conditions (1) and (2) are not incompatible. Recall that the closed balls $\bar{B}\left(q_{i}, r_{1}\right)$ are pairwise disjoint (cf. Claim), so the choice of such an $\epsilon>0$ is possible.

Let us assume for the moment that a smooth quasiconformal mapping $F_{0}$ : $A_{0}^{\epsilon} \rightarrow \tilde{A}_{0}^{\epsilon}$, satisfying properties (1) and (2), exists. We define $F_{n}: A_{(n)}^{\epsilon} \rightarrow \tilde{A}_{(n)}^{\epsilon}$ such that $\left.F_{n}\right|_{A_{i_{1} \ldots i_{n}}^{\epsilon}}: A_{i_{1} \ldots i_{n}}^{\epsilon} \rightarrow \tilde{A}_{i_{1} \ldots i_{n}}^{\epsilon}$ by the formula

$$
\left.F_{n}\right|_{A_{i_{1} \ldots i_{n}}^{e}}=g_{i_{n}}^{-1} \circ \cdots \circ g_{i_{1}}^{-1} \circ F_{0} \circ f_{i_{1}} \circ \cdots \circ f_{i_{n}} \text { on } A_{i_{1} \ldots i_{n}}^{\epsilon} \text {. }
$$

We use $F_{n}$ to define our mapping $F: H^{1} \backslash S_{\beta} \rightarrow H^{1} \backslash S_{\alpha}$ by $\left.F\right|_{A_{i_{1} \ldots i_{n}}}=F_{n}$. For the correctness of this definition we have to check that

$$
F_{n}=F_{n-1} \quad \text { on } R_{(n)}^{\epsilon}=A_{(n)}^{\epsilon} \cap A_{(n-1)}^{\epsilon} .
$$

Observe that $R_{(n)}^{\epsilon}$ is a union of the spherical rings of the form

$$
R_{i_{1} \ldots i_{n}}^{\epsilon}:=f_{i_{n}}^{-1} \circ \cdots \circ f_{i_{1}}^{-1} R_{0}^{\epsilon}
$$

Using the definition (3.2) and property (2) of $F_{0}$, we obtain that the right side of (3.3) will be

$$
\left.F_{n-1}\right|_{R_{i_{1} \ldots i_{n}}^{\epsilon}}=g_{i_{n}}^{-1} \circ \cdots \circ g_{i_{1}}^{-1} \circ f_{i_{1}} \circ \cdots \circ f_{i_{n}} .
$$

Similarly, using property (1) of $F_{0}$, we obtain the same expression for the left side of (3.3). In conclusion, our mapping $F: H^{1} \backslash S_{\beta} \rightarrow H^{1} \backslash S_{\alpha}$ is well-defined.

Let us now recall that $F_{0}: A_{0}^{\epsilon} \rightarrow \tilde{A}_{0}^{\epsilon}$ is a $K$-quasiconformal mapping for a certain $K=K(\alpha, \beta)>1$. Furthermore, $\left.F\right|_{A_{(n)}^{\epsilon}}=F_{(n)}$; and by (3.2) we have that $F_{(n)}=g_{(n)}^{-1} \circ F_{0} \circ f_{(n)}$, where $g_{(n)}$ and $f_{(n)}$ are finite compositions of translations and dilations. Composition with $f_{(n)}$ and $g_{(n)}$ does not change the quasiconformal distortion, so it follows that $F$ is $K$-quasiconformal on $H^{1} \backslash S_{\beta}$.

One can see directly from the definition that $F: H^{1} \backslash S_{\beta} \rightarrow H^{1} \backslash S_{\alpha}$ has a homeomorphic extension $\tilde{F}: H^{1} \rightarrow H^{1}$ such that $\tilde{F} S_{\beta}=S_{\alpha}$. We now use Theorem 1.3 from [BaKo] to conclude that the extension $\tilde{F}: H^{1} \rightarrow H^{1}$ is a $K_{1}$-quasiconformal mapping for some $K_{1} \geq K$. 
It remains to construct the mapping $F_{0}: A_{0}^{\epsilon} \rightarrow \tilde{A}_{0}^{\epsilon}$, satisfying properties (1) and (2). This is done by the flow method of Koranyi and Reimann. Namely, we consider the vector-field of the form

$$
V:=p \cdot T+\frac{1}{4}[(X p) Y-(Y p) X],
$$

where $X$ and $Y$ are the left-invariant vector-fields from (2.4), $T=\partial / \partial z$ and $p$ is a smooth, compactly supported function. We consider the flow $\left(F_{s}\right)_{s \in \mathbf{R}}$ generated by the vector field $V$. It follows by Section 5 of [KoRe2] that, for each $s \in \mathbf{R}$, the mapping $F_{s}: H^{1} \rightarrow H^{1}$ is a $C^{\infty}$ smooth QC mapping.

Our map $F_{0}: A_{0}^{\epsilon} \rightarrow \tilde{A}_{0}^{\epsilon}$ will be defined as the restriction to $A_{0}^{\epsilon}$ of a time-s map $F_{s}: H^{1} \rightarrow H^{1}$ as above. The generating function $p: H^{1} \rightarrow \mathbf{R}$ in (3.4) is defined in a special way to ensure (1) and (2). To do this, it is convenient to reduce the problem to a simpler situation. Namely, let $q_{0} \in H^{1}$ be a point and let $0<r_{2}<r_{1}<r_{0}$. We shall define a function $p_{0}: H^{1} \rightarrow \mathbf{R}$ with the following properties:

(a) supp $p_{0} \subset B\left(q_{0}, r_{0}\right)$,

(b) $F_{0 s} B\left(q_{0}, r_{1}\right) \subset B\left(q_{0}, r_{1}\right)$ for all $s \geq 0$,

(c) there exists $s=s\left(r_{2}, r_{1}\right)$ such that $\left.F_{0 s}\right|_{B\left(q_{0}, r_{1}\right)}=T_{q_{0}} \circ \delta_{r_{2} / r_{1}} \circ T_{q_{0}^{-1}}$.

Here $F_{0 s}$ stands for the time-s map of the vector field $V_{0}$ given by (3.4) with the generating function $p_{0}$.

Let us assume that for given data $q_{0} \in H^{1}, 0<r_{2}<r_{1}<r_{0}$, we have already constructed a smooth function $p_{0}=p_{0}\left(q_{0}, r_{2}, r_{1}, r_{0}\right)$ ensuring properties (a), (b) and (c). Let us return to our balls $\left\{B\left(q_{i}, r_{1}\right)\right\}_{i=1}^{N}$ and dynamics $f: \bigcup_{i} B\left(q_{i}, r_{1}\right) \rightarrow B$. Choose $\epsilon>0$ so small that, in addition to (3.1), we also have

$$
B\left(q_{i}, r_{1}+2 \epsilon\right) \subset B(0,1-\epsilon)
$$

and

$$
B\left(q_{i}, r_{1}+2 \epsilon\right) \cap B\left(q_{j}, r_{1}+2 \epsilon\right)=\emptyset \quad \text { for } i \neq j .
$$

Let $p_{i}: H^{1} \rightarrow \mathbf{R}$ be the smooth functions associated to data $q_{i}, 0<r_{2}<r_{1}+\epsilon<$ $r_{1}+2 \epsilon$ as above and define $p: H^{1} \rightarrow \mathbf{R}, p=\sum_{i=1}^{N} p_{i}$. It follows that the time- $s$ map $F_{s}: H^{1} \rightarrow H^{1}$ of the vector field associated by (3.4) to $p$ satisfies

$$
F_{s}=\mathrm{id} \quad \text { on } H^{1} \backslash\left(\bigcup_{i} B\left(q_{i}, r_{1}+2 \epsilon\right)\right),
$$

and there exists $s=s\left(r_{2}, r_{1}\right)$ such that

$$
\left.F_{s}\right|_{B\left(q_{i}, r_{1}+\varepsilon\right)}=T_{q_{i}} \circ \delta_{r_{2} / r_{1}} \circ T_{q_{i}^{-1}}=g_{i}^{-1} \circ f_{i} \quad \text { for all } i=1, \ldots, N \text {. }
$$

Defining $F_{0}:=\left.F_{s}\right|_{A_{0}^{\varepsilon}}$ for $s=s\left(r_{2}, r_{1}\right)$, we see that properties (1) and (2) are implied by (3.5) and (3.6). 
Our final task is to define $p_{0}$ ensuring properties (a), (b), (c) for arbitrary data $q_{0}=\left(x_{0}, y_{0}, z_{0}\right), 0<r_{2}<r_{1}<r_{0}$. Let $a=r_{2} / r_{1}<1$ and let us write an arbitrary point $q \in H^{1}$ as

$$
q=q_{0} * q_{1}=\left(x_{0}+x_{1}, y_{0}+y_{1}, z_{0}+z_{1}+2\left(y_{0} x_{1}-x_{0} y_{1}\right)\right)
$$

Then we have

$$
\begin{aligned}
\left(T_{q_{0}} \circ \delta_{a} \circ T_{q_{0}^{-1}}\right) q & =T_{q_{0}} \delta_{a} q_{1} \\
& =\left(x_{0}+a x_{1}, y_{0}+a y_{1}, z_{0}+a^{2} z_{1}+2 a\left(\left(y_{0} x_{1}-y_{1} x_{0}\right)\right) .\right.
\end{aligned}
$$

Let us first consider the auxiliary function $\tilde{p}_{0}: H^{1} \rightarrow \mathbf{R}$

$$
\tilde{p}_{0}(x, y, z)=y_{0} x-x_{0} y-\frac{1}{2}\left(z-z_{0}\right) \text {. }
$$

By (3.4), the associated vector field will be

$$
\tilde{V}_{0}=-\frac{1}{4}\left(x-x_{0}\right) \frac{\partial}{\partial x}-\frac{1}{4}\left(y-y_{0}\right) \frac{\partial}{\partial y}+\frac{1}{2}\left[y_{0} x-x_{0} y-z+z_{0}\right] \frac{\partial}{\partial z} .
$$

The flow $\tilde{F}_{0 s}$ of $\tilde{V}_{0}$ is given by the solution of the linear system of ODE

$$
\left\{\begin{array}{l}
\dot{x}=-\frac{1}{4}\left(x-x_{0}\right) \\
\dot{y}=-\frac{1}{4}\left(y-y_{0}\right) \\
\dot{z}=\frac{1}{2}\left[y_{0} x-x_{0} y-z+z_{0}\right]
\end{array}\right.
$$

with initial conditions

$$
(x(0), y(0), z(0))=\left(x_{0}+x_{1}, y_{0}+y_{1}, z_{0}+z_{1}+2\left(y_{0} x_{1}-x_{0} y_{1}\right)\right) .
$$

Solving (3.8) with the above initial conditions, we obtain

$$
\left\{\begin{array}{l}
x(s)=x_{0}+x_{1} e^{-\frac{1}{4} s} \\
y(s)=y_{0}+y_{1} e^{-\frac{1}{4} s} \\
z(s)=z_{0}+z_{1} e^{-\frac{1}{2} s}+2\left(y_{0} x_{1}-y_{1} x_{0}\right) e^{-\frac{1}{4} s}
\end{array}\right.
$$

From (3.9), we see immediately that the image $\tilde{F}_{0 s}(q)$ of $q=q_{0} * q_{1}$ under the flow $\left(\tilde{F}_{0 s}\right)_{s}$ is given by

$$
\tilde{F}_{0 s}(q)=T_{q_{0}} \circ \delta_{e^{-s / 4}} \circ T_{q_{0}^{-1}}(q) .
$$

It is clear that for $s \geq 0$, we have $\tilde{F}_{0 s} B\left(q_{0}, r_{1}\right) \subseteq B\left(q_{0}, r_{1}\right)$; and for the value

$$
s=-4 \log \frac{r_{2}}{r_{1}}, \quad \text { we have } \tilde{F}_{0 s}=T_{q_{0}} \circ \delta_{r_{2} / r_{1}} \circ T_{q_{0}^{-1}} .
$$


We now obtain $p_{0}$ by multiplying $\tilde{p}_{0}$ by a cutoff function $\eta: H^{1} \rightarrow \mathbf{R}$ such that $\eta \equiv 1$ on $B\left(q_{0}, r_{1}\right)$ and supp $\eta \subset B\left(q_{0}, r_{0}\right)$. For the function $p_{0}=\eta \tilde{p}_{0}$, the required properties (a), (b), and (c) clearly follow. This concludes the proof of Theorem 1.1.

A case of special interest is when our Cantor-type sets lie on the $O z$ axis. For this, let us consider the above construction with $q_{1}=(0,0,1 / 2)$, $q_{2}=(0,0,-1 / 2)$ and choose $r_{1}=a / \sqrt{2}$. One easily checks that for $a<1$ we have $B\left(q_{1}, a / \sqrt{2}\right) \cap B\left(q_{2}, a / \sqrt{2}\right)=\emptyset$ and $B\left(q_{i}, a / \sqrt{2}\right) \subset B$ for $i=1,2$. The corresponding dynamical system $f: \bigcup_{i=1}^{2} B\left(q_{i}, a / \sqrt{2}\right) \rightarrow B$ has an invariant set $S=S(a)$ which is contained in the $O z$ axis. This follows from the fact that the whole $O z$ axis is invariant under both $f_{1}$ and $f_{2}$.

According to (2.9), the Hausdorff dimension of $S(a)$ is given by

$$
\operatorname{dim} S(a)=\frac{2 \log 2}{\log 2-2 \log a} .
$$

This implies that $\operatorname{dim} S(a) \rightarrow 2$ as $a \rightarrow 1$ and $\operatorname{dim} S(a) \rightarrow 0$ as $a \rightarrow 0$. The proof of Theorem 1.1 gives the following.

Corollary 3.1. For any two values $0<\alpha<\beta<2$, there exist sets $S_{\alpha}, S_{\beta}$ and a quasiconformal mapping $F: H^{1} \rightarrow H^{1}$ such that $S_{\alpha} \subset O z, S_{\beta} \subset O z, \operatorname{dim} S_{\alpha}=$ $\alpha, \operatorname{dim} S_{\beta}=\beta$ and $F S_{\beta}=S_{\alpha}$. If $\alpha<1$, we can choose $S_{\alpha}$ to be a subset of $a$ rectifiable curve.

Proof of Corollary 3.1. The first statement of the Corollary follows directly from the proof of Theorem 1.1. We prove here the second statement.

By (3.10), $\operatorname{dim} S(a)<1$ iff $a<1 / \sqrt{2}$. We show that in this case $S(a) \subset \Gamma$, where $\Gamma$ is a curve that is rectifiable with respect to Heisenberg metric. To construct $\Gamma$, we use the dynamics $f: \bigcup_{i=1}^{2} B_{i} \rightarrow B$. Consider the two points $Q_{1}=(0,0,1), Q_{2}=$ $(0,0,-1)$ and let $Q_{1}^{\prime}=f_{1}^{-1} Q_{1}, Q_{2}^{\prime}=f_{2}^{-1} Q_{2}$. Then $Q_{i}^{\prime} \in \partial B_{i}$ for $i=1,2$.

Choose smooth Legendrian curves $\Gamma_{0}^{i} \subset \bar{B} \backslash\left(\bigcup_{i=1}^{2} B_{i}\right)$ connecting $Q_{i}$ and $Q_{i}^{\prime}$ for $i=1,2$. A Legendrian curve is a smooth regular curve whose tangent is in $\operatorname{span}\{X, Y\}$, where $X, Y$ are the vector-fields given in (2.4). For any two points in a domain $D \subseteq H^{1}$, there always exists a Legendrian curve in $D$ connecting the two points. This follows easily from Chow's theorem (cf. [Be]).

Similarly, let $Q_{1}^{\prime \prime}=f_{2}^{-1} Q_{1}, Q_{2}^{\prime \prime}=f_{1}^{-1} Q_{2}$ and let $\Gamma_{0}^{3}$ connect $Q_{1}^{\prime \prime}$ and $Q_{2}^{\prime \prime}$ in $B \backslash\left(\bigcup_{i=1}^{2} B_{i}\right)$. Denote by $\Gamma_{0}:=\bigcup_{i=1}^{3} \Gamma_{0}^{i}$. Then $\Gamma_{0} \subset B \backslash\left(\bigcup_{i=1}^{2} B_{i}\right)$, and

$$
\text { length } \Gamma_{0}=\sum_{i=1}^{3} \text { length } \Gamma_{0}^{i}=l_{0} \text {. }
$$


Here and in the following, the length of a curve is measured in the Heisenberg metric. Let us denote by $\Gamma_{n}$ the $n$-th preimage of $\Gamma_{0}$ under $f, \Gamma_{n}:=f^{-n} \Gamma_{0}$, for each $n>0$. Then $\Gamma_{n}$ consists of $3 \cdot 2^{n}$ pieces of smooth Legendrian curves with total length

$$
l_{n}=2^{n}(a / \sqrt{2})^{n} l_{0}=(\sqrt{2} a)^{n} l_{0} .
$$

We define $\Gamma:=\overline{\bigcup_{n \geq 0} \Gamma_{n}}$. We leave to the reader to check that $S(a) \subset \Gamma$ and that $\Gamma$ is a rectifiable curve with

$$
\text { length } \Gamma=\sum_{n} l_{n}=\frac{l_{0}}{1-\sqrt{2} a} .
$$

Remark. A question of Heinonen and Semmes (see [HeSe]) asks whether there exists a quasiconformal mapping of the Heisenberg group that maps the $O z$ axis onto a rectifiable curve. The above Corollary shows that certain subsets of $\mathrm{Oz}$ with Hausdorff dimension arbitrarily close to 2 can be mapped into a rectifiable curve.

\section{Bounds on dimension distortion}

In this section, we work in the general setting of a metric measure space $(X, d, \mu)$. We assume that $(X, d)$ is a proper, unbounded metric space and $\mu$ is a Borel measure on $X$ that is $Q$-regular for some $Q \geq 1$. This means that there exists a constant $C=C(X) \geq 1$ such that for any ball $B(p, r) \subset X$ we have

$$
\frac{1}{C} r^{Q} \leq \mu(B(p, r)) \leq C r^{Q}
$$

In addition, we assume that $X$ supports a weak $(1, q)$-Poincaré inequality for some $q<Q$. We refer to the paper of Heinonen and Koskela [HeKo] for the definition and a thorough treatment of the QC theory on metric spaces supporting a weak Poincare inequality. This framework is quite general and allows spaces with fractal character or sub-Riemannian geometry. For example, the Heisenberg group $H^{1}$ is 4-regular and supports a weak $(1, q)$-Poincaré inequality for any $q \geq 1$ by a result of Jerison [Je].

The content of the results from [HeKo] is that most of the classical theory of QC maps in $\mathbf{R}^{n}$ remain valid in this abstract setting. Similarly to the definition from (1.1), we say that a homeomorphism $f: X \rightarrow X$ is a $K-\mathrm{QC}$ map if

$$
H(x, f):=\lim \sup _{r \rightarrow 0} \frac{\sup _{d(x, y) \leq r} d(f x, f y)}{\inf _{d(x, y) \geq r} d(f x, f y)} \leq K, \quad \text { for all } x \in X .
$$


In this section, we consider the following problem. Let us denote by $\mathcal{F}(X, K)$ the class of homeomorphisms $f: X \rightarrow X$ that map bounded sets to bounded sets and are $K$-quasiconformal for a fixed $K \geq 1$. Given a set $A \subset X$ with Hausdorff dimension $\operatorname{dim} A \leq \alpha$, we are interested in estimating the greatest value $\beta=\beta(X, K, \alpha)$ of the Hausdorff dimension of the image set $\operatorname{dim} f A$ for $f \in \mathcal{F}(X, K)$ :

$$
\beta=\beta(X, K, \alpha)=\sup \{\operatorname{dim} f A: \operatorname{dim} A \leq \alpha, f \in \mathcal{F}(X, K)\} .
$$

Let us fix $K \geq 1$ and consider $f \in \mathcal{F}(X, K)$. From Section 4 of [HeKo] (see also [BaKo]), it follows that $f$ is quasisymmetric. This is a global property, which means that there exists a homeomorphism $\eta:[0, \infty) \rightarrow[0, \infty)$ such that

$$
d(x, y) \leq t d(x, z) \text { implies } d(f x, f y) \leq \eta(t) d(f x, f z),
$$

for all $x, y, z \in X, t>0$. Moreover, the quasisymmetry function $\eta$ depends only on $X$ and $K$ (and not on $f$ ).

Heinonen and Koskela (Theorem 7.11 [HeKo]) proved that $\eta$-quasisymmetric mappings have a remarkable higher integrability property. This generalizes a classical result of Gehring [Ge] from the Euclidean case. More exactly, if $f$ : $X \rightarrow X$ is $\eta$-quasisymmetric, then there is an exponent $r=r(X, \eta)>1$ such that $J_{f} \in L_{l o c}^{r}$, where $J_{f}$ is the volume derivative of $f$ :

$$
J_{f}(x)=\lim _{r \rightarrow 0} \frac{\mu(f B(x, r))}{\mu(B(x, r))} \quad \text { for } \mu \text { a.e. } x \in X .
$$

Let us introduce the exponent of integrability for the class $\mathcal{F}(X, K)$ :

$$
p=p(X, K)=\sup \left\{r>0: J_{f} \in L_{\text {loc }}^{r} \text { for any } f \in \mathcal{F}(X, K)\right\} .
$$

The result of this section is the following abstract analogue of Theorem 12 in [GeVa]:

Theorem 4.1. Let $X$ be an unbounded, proper, $Q$-regular metric space supporting a weak $(1, q)$-Poincaré inequality for some $q<Q$. Let $0 \leq \alpha \leq Q$ and $A \subset X$ be a set of Hausdorff dimension $\operatorname{dim} A \leq \alpha$. Then for any $f \in \mathcal{F}(X, K)$, the image set $f A$ has Hausdorf dimension

$$
\operatorname{dim} f A \leq \frac{Q p \alpha}{Q(p-1)+\alpha},
$$

where $p=p(X, K)$ is the exponent of integrability from (4.2). 
Proof of Theorem 4.1. We may clearly assume that our set $A$ is contained in a large ball $B_{0} \subset X$.

The case $\alpha=Q$ is obvious. We prove the statement for $\alpha<Q$. For each $a \in$ $(\alpha, Q)$ and each $r \in(1, p)$, we show that the outer Hausdorff measure $H_{b}(f A)=0$, where

$$
b=\frac{Q r a}{Q(r-1)+a} .
$$

We cannot use in the proof non-overlapping squares as in the Euclidean case (cf. [GeVa]); our argument is based on the $5 r$-covering theorem (see p. 24 of [Ma]).

Let us choose an arbitrary $\epsilon>0$ and $d>0$. Because $H_{a}(A)=0$, we can find a countable collection $\left\{B_{j}\right\}_{j}$ of balls $B_{j}=B\left(x_{j}, r_{j}\right)$ such that

(a) $A \subset \bigcup_{j} B_{j} \subset B_{0}$,

(b) $\operatorname{diam} f B_{j}<d$, and

(c) $\sum_{j} r_{j}^{a}<\epsilon$.

Using the $5 r$-covering theorem, we can select a subcollection $\left\{B_{i}\right\}_{i}$ of the collection $\left\{B_{j}\right\}_{j}$ such that

(a) $A \subset \bigcup_{i} B_{i} \subset B_{0}$,

(b) $\operatorname{diam} f B_{i}<d$,

(c) $\sum_{i} r_{i}^{a}<\epsilon$, and

(d) $\frac{1}{5} B_{i_{1}} \cap \frac{1}{5} B_{i_{2}}=\emptyset$ for $i_{1} \neq i_{2}$.

We write

$$
L_{i}=L_{f}\left(x_{i}, r_{i}\right)=\sup \left\{d\left(f x, f x_{i}\right): d\left(x_{i}, x\right) \leq r_{i}\right\}
$$

and

$$
l_{i}=l_{f}\left(x_{i}, \frac{1}{5} r_{i}\right)=\inf \left\{d\left(f x, f x_{i}\right): d\left(x_{i}, x\right) \geq \frac{1}{5} r_{i}\right\} .
$$

It is clear that $l_{i} \leq L_{i}$. Since $f$ is quasisymmetric, we can apply (4.1) to obtain $L_{i} \leq \eta(5) l_{i}$. Consequently, $L_{i} \leq C l_{i}$ for some uniform constant $C=C(X, K) \geq 1$. In what follows, $C=C(X, K)>0$ will denote an absolute, generic constant whose value can change even within one string of inequalities.

The relation $L_{i} \leq C l_{i}$ together with $Q$-regularity yields

$$
\operatorname{diam} f B_{i} \leq 2 L_{i} \leq C l_{i} \leq C\left(\mu B\left(f x_{i}, l_{i}\right)\right)^{1 / Q} \leq C\left(\mu f\left(\frac{1}{5} B_{i}\right)\right)^{1 / Q} .
$$

Because $f$ is absolutely continuous in measure (see [HeKo] Corollary 7.13), it follows by Hölder's inequality and Q-regularity that

$$
\mu\left(f\left(\frac{1}{5} B_{i}\right)\right)=\int_{\frac{1}{b} B_{i}} J_{f} d \mu \leq C r_{i}^{Q \frac{r-1}{r}}\left(\int_{b^{3} B_{i}}\left(J_{f}\right)^{r} d \mu\right)^{\frac{3}{r}} .
$$


From (4.3) and (4.4), we obtain

$$
\sum_{i}\left[\operatorname{diam}\left(f B_{i}\right)\right]^{b} \leq C \sum_{i} r_{i}^{b \frac{r-1}{r}}\left(\int_{\frac{1}{5} B_{i}}\left(J_{f}\right)^{r} d \mu\right)^{\frac{b}{Q r}} .
$$

Since $\left\{f B_{i}\right\}_{i}$ is a covering of $f A$, relation (4.5) implies a good estimate for $H_{\dot{b}}(f A)$ via Hölder's inequality:

$$
\sum_{i}\left[\operatorname{diam}\left(f B_{i}\right)\right]^{b} \leq C\left(\sum_{i} r_{i}^{a}\right)^{\frac{b(r-1)}{a r}}\left(\sum_{i} \int_{\frac{1}{5} B_{i}}\left(J_{f}\right)^{r} d \mu\right)^{\frac{b}{Q r}} .
$$

Relation (4.6) together with properties (c) and (d) of our covering imply

$$
\sum_{i}\left[\operatorname{diam}\left(f B_{i}\right)\right]^{b} \leq C \epsilon^{\frac{b(r-1)}{a r}}\left(\int_{B_{0}}\left(J_{f}\right)^{r} d \mu\right)^{\frac{b}{Q r}} .
$$

Letting $\epsilon \rightarrow 0$, we obtain $H_{b}(f A)=0$ as required.

Theorem 4.1 gives the upper estimate of Theorem 1.2. For the lower estimate, notice that in $Q$-regular spaces $X$ admitting an $(1, q)$-Poincaré inequality with $q<Q$, a $K$-QC mapping $f$ is quasisymmetric. The inverse of a quasisymmetric mapping is also quasisymmetric, thus quasiconformal. However, the constant of quasiconformality of $f^{-1}$ is $K^{\prime}$; and it could be that $K^{\prime}>K$. On the Heisenberg group, the situation is better: the inverse of a $K$-quasiconformal mapping is also $K$-quasiconformal by Proposition 20 in [KoRe2]. Thus Theorem 4.1 also implies the lower estimate in Theorem 1.2.

\section{Remarks.}

1. It is an interesting problem for further research to determine the sharp value of the exponent of integrability $p\left(H^{1}, K\right)$ from (1.2). The same problem is difficult even in the Euclidean case. The only exact result in this direction is in $\mathbf{R}^{2}$; it is due to Astala [As], who showed that $p\left(\mathbf{R}^{2}, K\right)=K /(K-1)$.

2. In the case when $X$ supports a $(1, Q)$ Poincaré inequality only (cf. [HeKo]), it is not known whether the higher integrability result still holds.

\section{Minimal-dimensional sets in the Heisenberg group}

In this section, we prove Theorem 1.3. Our task is to construct subsets of $H^{1}$ whose Hausdorff dimension cannot be lowered by QC maps.

Proof of Theorem 1.3. Given any number $1 \leq \alpha<4$, we have to construct a set $S_{\alpha}$ with $\operatorname{dim} S_{\alpha}=\alpha \operatorname{such}$ that $\operatorname{dim} f S_{\alpha} \geq \alpha$ for any QC map $f: H^{1} \rightarrow H^{1}$. We 
consider first the case $1<\alpha<3$. We use Pansu's notion of conformal dimension of a metric space $X$ defined by

$$
\operatorname{dim}_{\text {conf }} X:=\inf _{Y} \operatorname{dim} Y
$$

where the inf is taken over all metric spaces $Y$ that are quasisymmetrically equivalent to $X$. Two metric spaces $X$ and $Y$ are said to be quasisymmetrically equivalent if there exists a quasisymmetry $f: X \rightarrow Y$. For the definition of a quasisymmetry, see (4.1). A lower bound on the conformal dimension is given by

Lemma A. Let $X$ be a compact $\alpha$-regular metric space. Suppose that there exists a curve family $\Gamma$ in $X$ with the following properties:

(a) there exists $\delta>0$ such that $\operatorname{diam} \gamma>\delta$ for each $\gamma \in \Gamma$,

(b) there exists a constant $C<\infty$ and a probability measure $\tilde{\mu}$ on $\Gamma$ such that

$$
\tilde{\mu}\{\gamma \in \Gamma: \gamma \cap B \neq \emptyset\} \leq C r^{\alpha-1}
$$

for each ball $B$ in $X$ of radius $r$.

Then

$$
\operatorname{dim}_{\text {conf }} X \geq \alpha
$$

Remark. Lemma A follows from Lemme 1.6 in [Bo] (see also Lemma 3.9 in [Ty]). The basic idea behind this statement goes back to Pansu (cf. Lemme 6.3 in [Pa1]).

Since a quasiconformal mapping $f: H^{1} \rightarrow H^{1}$ is always quasisymmetric, we can apply Lemma $\mathrm{A}$, once we construct an $\alpha$-regular subset $X \subset H^{1}$ satisfying conditions (a) and (b).

The set $X=\tilde{S}_{\alpha}$ will be of product-type and foliated by horizontal line segments in the $0 x z$ plane emerging from a Cantor set $S_{\alpha-1} \subset O z$ with $\operatorname{dim} S_{\alpha-1}=\alpha-1$, $0<\alpha-1<2$. Such a set $S_{\alpha-1}$ has already been constructed in the proof of Corollary 3.1. Recall also from Section 2 that $S_{\alpha-1}$ carries an $(\alpha-1)$-regular probability measure $\mu$ : there exists $C \geq 1$ such that for any $r \leq 1, p \in S_{\alpha-1}$ we have

$$
\frac{1}{C} r^{\alpha-1} \leq \mu\left(B(p, r) \cap S_{\alpha-1}\right) \leq C r^{\alpha-1}
$$

The set $\tilde{S}_{\alpha}$ is defined by

$$
\tilde{S}_{\alpha}=\left\{T^{s}(p):=(s, 0,0) * p: p \in S_{\alpha-1}, s \in[0,1]\right\} .
$$


To show that $\tilde{S}_{\alpha}$ is an $\alpha$-regular set, we construct a probability measure $\nu$ on $\tilde{S}_{\alpha}$ such that for any $q \in \tilde{S}_{\alpha}, r \leq 1$,

$$
\frac{1}{C} r^{\alpha} \leq \nu\left(B(q, r) \cap \tilde{S}_{\alpha}\right) \leq C r^{\alpha},
$$

for some uniform constant $C \geq 1$. The measure $\nu$ is defined as a product measure $\nu=\mu * m$, where $m$ is the usual 1-dimensional Lebesgue measure on [0,1]. More precisely, for sets of product-type

$$
A * I=\left\{T^{s}(p): p \in A \subset S_{\alpha-1}, s \in I \subset[0,1]\right\},
$$

where $A \subset S_{\alpha-1}, I \subset[0,1]$ are Borel subsets, we define $\nu$ by

$$
\nu(A * I)=\mu(A) m(I)
$$

and extend it to a measure on the $\sigma$-algebra generated by sets of type $A * I$.

To prove (5.3), we introduce some notation. For $C \geq 1, r, s \in[0,1]$, denote by $I(C, r, s)$ the interval $[s, s+C r]$. Relation (5.3) follows now from (5.1), (5.4) and the following

Claim 1. There exists $C \geq 1$ such that for any $p \in S_{\alpha-1}, s, r \in[0,1]$ we have the inclusions

$$
\left(B\left(p, \frac{1}{C} r\right) \cap S_{\alpha-1}\right) * I\left(\frac{1}{C}, r, s\right) \subseteq B\left(T^{s}(p), r\right) \cap \tilde{S}_{\alpha} \subseteq\left(B(p, C r) \cap S_{\alpha-1}\right) * I(C, r, s)
$$

Proof of Claim 1. Because $T^{s}: H^{1} \rightarrow H^{1}$ is an isometry for any $s \in[0,1]$, it is enough to prove Claim 1 for $s=0$. Let us show the right inclusion. Choose an arbitrary point $x \in B(p, r) \cap \tilde{S}_{\alpha}$. Then $x=T^{s_{1}}(q)$ for some $s_{1} \in[0,1], q \in S_{\alpha-1}$. Observe that $s_{1}=d_{H}(x, q)$. Similarly, a direct calculation gives that $d_{H}(x, q) \leq$ $d_{H}(x, p) \leq r$. This gives that $s_{1} \leq r$. By the triangle inequality, $d_{H}(p, q) \leq$ $d_{H}(x, q)+d_{H}(x, p) \leq 2 r$. In conclusion, $x=T^{s_{1}}(q) \in\left(B(p, 2 r) \cap S_{\alpha-1}\right) *[0, r]$, as required. The left inclusion is left as an exercise for the reader.

Let us denote by $\Gamma$ the curve family

$$
\Gamma=\left\{\gamma_{p}\right\}_{p \in S_{\alpha-1}}, \quad \gamma_{p}:[0,1] \rightarrow H^{1}, \quad \gamma_{p}(s)=T^{s}(p)
$$

The measure $\mu$ is transported from $S_{\alpha-1}$ to a probability measure $\tilde{\mu}$ on $\Gamma$. By the right inclusion from Claim 1, condition (b) of Lemma A follows directly. This proves Theorem 1.3 for $1<\alpha<3$.

For the values $3<\alpha<4$, we cannot use the same proof. The reason for this is that for higher dimensions we cannot construct a set with a nice fibration 
by horizontal curves where the uniform product structure guaranteed by Claim 1 holds. In this case, we use a different argument.

Let us start with some notation and terminology. Let $X \subset Y$ be a subset of the metric space $Y$. In what follows, denote the Hausdorff dimension of $X$ by $\operatorname{dim}_{H} X$. The $a$-dimensional Hausdorff content $H_{a}^{\infty}(X)$ of $X$ is defined by

$$
H_{a}^{\infty}(X)=\inf _{\mathcal{B}} \sum_{B_{i} \in \mathcal{B}}\left(\operatorname{diam} B_{i}\right)^{a},
$$

where the inf is taken over all countable coverings $\mathcal{B}=\left(B_{i}\right)_{i}$ of the set $X$. Observe that for bounded sets $H_{a}^{\infty}(X)$ is always finite, and we have $H_{a}^{\infty}(X) \leq H_{a}(X)$. Moreover, $H_{a}^{\infty}(X)=0$ iff $H_{a}(X)=0$.

We also consider the upper Minkowski dimension of $X$ given by

$$
\overline{\operatorname{dim}_{M}} X=\inf \left\{s>0: \lim \sup _{\epsilon \rightarrow 0} N(X, \epsilon) \epsilon^{s}=0\right\},
$$

where

$$
N(X, \epsilon)=\min \left\{k: X \subset \bigcup_{i=1}^{k} B\left(x_{i}, \epsilon\right), x_{i} \in X\right\} .
$$

For a general set $X$,

$$
\operatorname{dim}_{H} X \leq \overline{\operatorname{dim}_{M}} X,
$$

where equality holds in (5.5) if $X$ is a regular set. For the proof of (5.5) in the case when $X \subset \mathbf{R}^{n}$, we refer to Chapter 5 in [Ma]. The same proof also works for general metric spaces.

In the case $3<\alpha<4$, the set $S_{\alpha}=X$ will be a Cantor set of Heisenberg spheres defined as follows. Let $A \subset[1,2]$ be a regular Cantor set of (Euclidean) dimension $a, 0<a<1$. Let $S_{r}=\partial B(0, r)$ be the Heisenberg sphere of radius $r$ and define $X:=\bigcup_{r \in A} S_{r}$. The proof of Theorem 1.3 proceeds now with the following two statements.

\section{Lemma 5.1.}

$$
\operatorname{dim}_{H} X=\overline{\operatorname{dim}_{M}} X=3+a .
$$

Lemma 5.2. For any $Q C$ mapping $f: H^{1} \rightarrow H^{1}, \operatorname{dim}_{H} f X \geq 3+a$.

Proof of Lemma 5.1. Because of (5.5), it is enough to prove the following inequalities:

$$
\widetilde{\operatorname{dim}_{M}} X \leq 3+a
$$


and

$$
\operatorname{dim}_{H} X \geq 3+a .
$$

Relation (5.8) follows from the considerations in the proof of Lemma 5.2 (see Remark after (5.18) below). We prove (5.7). It suffices to show that there exists $C>0$ such that for $0<\epsilon \leq 1$ we have

$$
N(X, \epsilon) \leq C(1 / \epsilon)^{3+a} .
$$

For $0<\epsilon \leq 1$, let

$$
N(A, \epsilon)=\min \left\{j: A \subseteq \bigcup_{i=1}^{j}\left(r_{i}-\epsilon, r_{i}+\epsilon\right), r_{i} \in[1,2]\right\} .
$$

By the $a$-regularity of $A$, there exists $C \geq 1$ such that

$$
N(A, \epsilon) \leq C / \epsilon^{a} \text {. }
$$

Let $r_{1}, \ldots, r_{N(A, \epsilon)} \in[1,2]$ be such that $A \subset \bigcup_{i=1}^{N(A, \epsilon)}\left(r_{i}-\epsilon, r_{i}+\epsilon\right)$. Consider the spherical rings $R\left(r_{i}, \epsilon\right)=B\left(0, r_{i}+\epsilon\right) \backslash \vec{B}\left(0, r_{i}-\epsilon\right), i=1, \ldots, N(A, \epsilon)$. It follows that

$$
X \subset \bigcup_{i=1}^{N(A, \epsilon)} R\left(r_{i}, \epsilon\right)
$$

Using (2.2), we can estimate the Euclidean volume of $R(r, \epsilon)$ by

$$
|R(r, \epsilon)|=c(r) \epsilon+o(\epsilon)
$$

where $1 / C \leq c(r) \leq C$ for some constant $C \geq 1$ and $r \in[1,2]$.

Let

$n(r, \epsilon)=\max \{j:$ there exist $j$ disjoint balls of radius $\epsilon / 5$ contained in $R(r, 100 \epsilon)\}$.

By the $5 r$-covering theorem, (2.3), and (5.12), we obtain

$$
N(R(r, \epsilon), \epsilon) \leq n(r, \epsilon) \leq C / \epsilon^{3} .
$$

By the inclusion (5.11) and the estimates (5.10) and (5.13), relation (5.9) follows.

Proof of Lemma 5.2. Fix a small $0<\eta<a / 2$ and consider an arbitrary covering $\left(B\left(y_{i}, r_{i}^{\prime}\right)\right)_{i}$ of $f X \subset H^{1}$. We show that there exist $c>0, \epsilon>0$ such that for $r_{i}^{\prime} \leq \epsilon$ we have

$$
\sum_{i}\left(r_{i}^{\prime}\right)^{3+a-\eta} \geq c
$$


By the $5 r$-covering Theorem, we can assume that $B\left(y_{i}, \frac{1}{5} r_{i}^{\prime}\right) \cap B\left(y_{j}, \frac{1}{5} r_{j}^{\prime}\right)=\emptyset$ for $i \neq j$.

Since $f$ is quasisymmetric, there exists a ball $B\left(y_{0}, r_{0}\right)$ such that $B\left(y_{0}, r_{0}\right) \subseteq$ $f(B(0, r))$ for all $r \in A$. An important point in the proof is the next claim.

Claim 2. There exists a constant $c_{0}=c_{0}\left(r_{0}\right)>0$ such that for each $r \in A$,

$$
H_{3}^{\infty}\left(f\left(S_{r}\right)\right) \geq c_{0}
$$

Proof of Claim 2. The proof is based on Pansu's isoperimetric inequality [Pa2] (see also [Gr] pp. 159-164), which states that if $\Omega$ is a bounded domain in $\mathbf{R}^{3}$ with piecewise $C^{2}$ smooth boundary, then

$$
H_{3}(\partial \Omega) \geq c|\Omega|^{3 / 4}
$$

for a certain absolute constant $c>0$. We remind the reader that in relations (5.15) and (5.16) the Hausdorff measures/contents are taken with respect to the Heisenberg metric.

There are two main differences between (5.15) and (5.16). The first difference is that we do not have a smoothness assumption on (5.15); the second is that we need a lower estimate for $H_{3}^{\infty}(\partial \Omega)$ rather than just $H_{3}(\partial \Omega)$. Nevertheless, we can reduce (5.15) to (5.16) by the following elegant argument communicated to the author by Pierre Pansu.

Let $\Omega_{r}=f(B(0, r))$ and consider a finite covering $\left(B\left(z_{i}, \rho_{i}\right)\right)_{i}$ of $\partial \Omega_{r}$. Introduce a new domain $\Omega_{r}^{\prime}:=\Omega_{r} \bigcup_{i} B\left(z_{i}, \rho_{i}\right)$. Note that the boundary $\partial \Omega_{r}^{\prime}$ is piecewise smooth and $\partial \Omega_{r}^{\prime} \subset \bigcup_{i} \partial B\left(z_{i}, \rho_{i}\right)$. Applying the isoperimetric inequality (6.16) to $\Omega_{r}^{\prime}$, we obtain

$$
0<c_{1} \leq\left|\Omega_{r}\right|^{3 / 4} \leq\left|\Omega_{r}^{\prime}\right|^{3 / 4} \leq C H_{3}\left(\partial \Omega_{r}^{\prime}\right) \leq C \sum_{i} H_{3}\left(\partial B\left(z_{i}, \rho_{i}\right)\right) \leq C \sum_{i} \rho_{i}^{3},
$$

which gives $\sum_{i} \rho_{i}^{3} \geq c_{0}>0$ for any finite covering $\left(B\left(z_{i}, \rho_{i}\right)\right)_{i}$ of the boundary $\partial \Omega_{r}$. If we have an infinite covering, we can use the compactness of $\partial \Omega_{r}$ to obtain a finite subcovering and conclude (5.15) in this case also.

Set $x_{i}=f_{i}^{-1} y_{i}$. By the quasisymmetry of $f^{-1}$, there exists a constant $C=C(f) \geq 1$ and radii $r_{i}>0$ such that

$$
B\left(x_{i}, r_{i}\right) \subseteq f^{-1}\left(B\left(y_{i}, \frac{1}{5} r_{i}^{\prime}\right)\right) \subseteq f^{-1}\left(B\left(y_{i}, r_{i}^{\prime}\right)\right) \subseteq B\left(x_{i}, C r_{i}\right)
$$

Let us note that while $\left(B\left(x_{i}, C r_{i}\right)\right)_{i}$ forms a covering of $X$, the closures of the smaller balls $\left(\bar{B}\left(x_{i}, \frac{1}{5} r_{i}\right)\right)_{i}$ are disjoint, i.e., $\left(\bar{B}\left(x_{i}, \frac{1}{5} r_{i}\right) \cap X\right)_{i}$ forms a packing of $X$. 
Let

$$
\chi_{i}(r)= \begin{cases}1, & \text { if } f\left(S_{r}\right) \cap B\left(y_{i}, r_{i}^{\prime}\right) \neq \emptyset \\ 0, & \text { otherwise. }\end{cases}
$$

Using (5.15), we have

$$
c_{0} \leq H_{3}^{\infty}\left(f\left(S_{r}\right)\right) \leq \sum_{i}\left(r_{i}^{\prime}\right)^{3} \chi_{i}(r),
$$

for each $r \in A$. Integrating (5.17) with respect to the measure $\mu$ on $A$ and using the $a$-regularity of $\mu$, we obtain

$$
\begin{aligned}
c_{0} & \leq \sum_{i}\left(r_{i}^{\prime}\right)^{3} \mu\left(\left\{r \in A: S_{r} \cap f^{-1}\left(B\left(y_{i}, r_{i}^{\prime}\right)\right) \neq \emptyset\right\}\right) \\
& \left.\leq \sum_{i}\left(r_{i}^{\prime}\right)^{3} \mu\left(\left\{r \in A: S_{r} \cap B\left(x_{i}, C r_{i}\right)\right) \neq \emptyset\right\}\right) \leq C \sum_{i}\left(r_{i}^{\prime}\right)^{3} r_{i}^{a} .
\end{aligned}
$$

Remark. When $f$ is the identity map, $r_{i}^{\prime}=r_{i}$; and (5.18) gives the uniform lower bound $\sum_{i} r_{i}^{3+a}>c$ for an arbitrary covering $\left(B\left(x_{i}, r_{i}\right)\right)_{i}$ of $X$. This implies that $\operatorname{dim}_{H} X \geq 3+a$, which is exactly relation (5.8) needed in the proof of Lemma 5.1 .

To continue the proof of Lemma 5.2, let us choose $b>1$ such that $b a>$ $\overline{\operatorname{dim}_{M}} X=3+a$. Since $\left(\bar{B}\left(x_{i}, \frac{1}{5} r_{i}\right) \cap X\right)_{i}$ forms a packing of $X$, it follows (see [Ma] or [Bo]) that there exists $\delta=\delta(b)>0$ such that $r_{i} \leq \delta$ implies that $\sum_{i} r_{i}^{b a} \leq 1$. Since $f^{-1}$ is uniformly continuous on compact sets, there exists $\epsilon>0$ such that if $r_{i}^{\prime} \leq \epsilon$, then $\operatorname{diam} f^{-1}\left(B\left(y_{i}, r_{i}^{\prime}\right)\right) \leq \delta$ and so $r_{i} \leq \delta$. The magnitude of $\epsilon>0$ depends only on $f$ and $\delta, \epsilon=\epsilon(f, \delta)$.

Applying Hölder's inequality to (5.18), we obtain

$$
0<c \leq\left(\sum_{i}\left(r_{i}^{\prime}\right)^{\frac{3 b}{b-1}}\right)^{\frac{b-1}{b}}\left(\sum_{i} r_{i}^{b a}\right)^{\frac{1}{b}} \leq\left(\sum_{i}\left(r_{i}^{\prime}\right)^{\frac{3 b}{b-1}}\right)^{\frac{b-1}{b}},
$$

under the condition $b>(3+a) / a$. Choosing $b=(3+a-\eta) /(a-\eta)>(3+a) / a$, we obtain (5.14) from (5.19). This completes the proof of Lemma 5.2.

To complete the proof of Theorem 1.3, it remains only to consider the cases $\alpha=1,3,4$, which are easy.

\section{REFERENCES}

[As] K. Astala, Area distortion of quasiconformal mappings, Acta Math. 173 (1994), 37-60.

[Ba] Z. M. Balogh, Equivariant contactomorphisms of circular surfaces, Houston J. Math. 25 (1999), 259-266. 
[BaKo] Z. M. Balogh and P. Koskela, Quasiconformality, quasisymmetry and removability in Loewner spaces, Duke Math. J. 101 (2000), 555-577.

[BaMi] A. Basmajian and R. Miner, Discrete subgroups of complex hyperbolic motions, Invent. Math. 131 (1998), 85-136.

[Be] A. Bellaïche, The tangent space in sub-Riemannian geometry, in Sub-Riemannian Geometry (A. Bellaïche and J.-J. Risler, eds.), Progress in Math. 144, Birkhăuser, Boston, 1996, pp. 4-78.

[Bi] C. J. Bishop, Quasiconformal mappings which increase dimension, Ann. Acad. Sci. Fenn. 24 (1999), 397-407.

[BiTy 1] C. J. Bishop and J. T. Tyson, Conformal dimension of the antenna set, preprint.

[BiTy2] C. J. Bishop and J. T. Tyson, Locally minimal sets for conformal dimension, Ann. Acad. Sci. Fenn. (to appear).

[Bo] M. Bourdon, Au bord de certains polyèdres hyperboliques, Ann. Inst. Fourier 45 (1995), 119-141.

[CaTa] L. Capogna and P. Tang, Uniform domains and quasiconformal mappings on the Heisenberg group, Manuscripta Math. 86 (1995), 267-281.

[Fa] K. Falconer, Fractal Geometry, Wiley, New York, 1990.

[Ge] F. W. Gehring, The $L^{p}$ integrability of the partial derivatives of a quasiconformal mapping, Acta Math. 130 (1973), 265-277.

[GeVa] F. W. Gehring and J. Văisălă, Hausdorff dimension and quasiconformal mappings, J. London Math. Soc. 6 (1973), 504-512.

[Gr] M. Gromov, Carnot-Carathéodory spaces seen from within, in Sub-Riemannian Geometry (A. Bellaïche and J.-J. Risler, eds.), Progress in Math. 144, Birkhăuser, Basel, 1996, pp. 79 323.

[GrPa] M. Gromov and P. Pansu, Rigidity of lattices: an introduction, in Geometric Topology: Recent Developments (Montecatini Terme, 1990), Lecture Notes in Math. 1504, Springer, Berlin, 1991, pp. 39-137.

[HeKo] J. Heinonen and P. Koskela, Quasiconformal maps in metric spaces with controlled geometry, Acta Math. 181 (1998), 1-61.

[HeSe] J. Heinonen and S. Semmes, Thirty-three yes or no questions about mappings, measures and metrics, Conform. Geom. Dynam. 1 (1997), 1-12.

[Je] D. Jerison, The Poincaré inequality for vector fields satisfying Hörmander's condition, Duke Math. J. 53 (1986), 503-523.

[Ko] A. Korányi, Geometric aspects of analysis on the Heisenberg group, in Topics in Modern Harmonic Analysis, Proc. Semin. Torino and Milano 1982, Vol. I, Ist. Naz. Alta Mat. Francesco Severi, Rome, 1983, pp. $209-259$.

[KoRe1] A. Korányi and H. M. Reimann, Quasiconformal mappings on the Heisenberg gnoup, Invent. Math. 80 (1985), 309-338.

[KoRe2] A. Korányi and H. M. Reimann, Foundations for the Theory of Quasiconformal Mappings on the Heisenberg Group, Advances in Math. 111 (1995), 1-87.

[Ma] P. Mattila, Geometry of Sets and Measures in Euclidean Spaces, Cambridge Studies in Advanced Mathematics, 1995.

[Mo] G. D. Mostow, Strong rigidity of locally symmetric spaces, Ann. of Math. Stud., No. 78, Princeton University Press, Princeton, N.J., University of Tokyo Press, Tokyo, 1973.

[Pa1] P. Pansu, Métriques de Carnot-Carathéodory et quasiisometries des espaces symétriques de rang un, Ann. Math. 129 (1989), 1-60.

[Pa2] P. Pansu, Une inégalité isopérimétrique sur le groupe d'Heisenberg, C. R. Acad. Sci. Paris 295 (1982), 127-131.

[Ri] S. Rickman, Personal communication. 
[Ta1] P. Tang, Quasiconformal homeomorphisms on CR 3-manifolds with symmetries, Math. Z. 219 (1995), 49-69.

[Ta2] P. Tang, Regularity and extremality of quasiconformal homeomorphisms on CR manifolds, Ann. Acad. Sci. Fenn. Ser. A I Math. 21 (1996), 289-308.

[Ty] J. T. Tyson, Sets of minimal Hausdorff dimension for quasiconformal maps, Proc. Amer. Math. Soc. (to appear).

\section{Zoltán M. Balogh}

MATHEMATISCHES INSTITUT

UNIVERSITÄT BERN

SIDLERSTR. 5

CH-3012 BERN, SWITZERLAND

email: zoltan@math-stat.unibe.ch

(Received December 15, 1999 and in revised form April 30, 2000) 